\title{
Fast-acting slow viruses
}

\section{Malcolm A. Martin}

IN the past seven years there has been a virtual explosion of new data about the human immunodeficiency viruses (HIVs) and their lentivirus relatives. Progress in molecular biology has been particularly impressive -- genomes have been sequenced, viral-encoded proteins have been identified, novel regulatory proteins and cis-acting elements have been elegantly dissected and, in a few instances, genetically engineered viral gene products have been inoculated into animals and man in attempts to elicit protective immunity. A considerable impediment to advances of equal importance in HIV biology and pathogenesis has been the lack of a tractable animal model. As a result, interest has shifted to the simian immunodeficiency viruses (SIVs) to provide answers to questions involving the life cycle of primate lentiviruses in vivo. Of particular note have been reports of molecular clones of SIV which direct the synthesis of replication-competent progeny virions. Unfortunately, none of these generate a virus capable of inducing disease in inoculated animals. Two reports, however, one by Kestler et al. (Desrosiers's group) in Science' and the other by Dewhurst et al. (Fultz's group) on page 636 of this issue ${ }^{2}$, describe the development of fatal disease in monkeys exposed to virus derived from new SIV clones. Experiments in vivo can now proceed full steam to evaluate the determinants of pathogenicity, the genomic variability, the role in vivo of some lentiviral accessory genes (for example, vif, $v p r, v p x$ and nef) and potentially useful antiviral agents or vaccines.

\section{SIV variant}

Simian immunodeficiency virus induces a syndrome in experimentally infected rhesus macaques that is remarkably similar to human AIDS. The animals develop an immunodeficiency state characterized by a loss of lymphocytes bearing the CD4 antigen, opportunistic infections, diarrhoea with severe weight loss, and central nervous system disease; most animals die between one and three years after infection. Kestler et al. ${ }^{1}$ used virus derived from a previously described SIV $_{\text {MAC }}$ clone $\left(\right.$ SIV $\left._{\text {MAC-239 }}\right)$ which replicates efficiently in peripheral blood mononuclear cells (PBMCs) of rhesus macaques. They observed a typical immunodeficiency syndrome and death in five of eleven monkeys during the first year of infection. The affected animals lost a significant amount of weight, became immunodeficient, and died with disseminated viral, bacterial or Pneumocystis infections.

Fultz and her colleagues previously identified an extremely virulent variant of SIV which, in contrast to SIV $_{\text {MAC }}$, causes an acute lethal disease in pig-tailed macaques as well as in other monkeys, with deaths occurring six to eight days after infection ${ }^{3}$. The variant, called SIV $_{\text {SMM-PBj } 14}$, arose spontaneously in a pig-tailed macaque originally infected with SIV isolated from a sooty mangabey monkey $\left(\mathrm{SIV}_{\mathrm{SMM}}\right)$. The pig-tailed macaques infected with SIV SMM-PBi14 $_{\text {devel- }}$ oped a profuse, bloody mucoid diarrhoea during the first week after infection and died one to three days later from severe depletion of body fluids and electrolytes. SIV $_{\text {SMM-PBj14 }}$ differed from its SIV $_{\text {SMM }}$ parent in several important ways: it had a wider host range (chimpanzee PBMCs and several continuous human $T$-lymphocyte lines), was cytopathic for sooty mangabey monkey PBMCs, and could not be neutralized in vitro with antibodies directed against SIV $_{\text {SMM }}$. Because of the fulminant and extremely rapid nature of $\mathrm{SIV}_{\mathrm{SMM} \text {-PBj14- }}$ induced disease and its primary effect on the gastrointestinal tract, the report by Fultz et al. was initially viewed with scepticism in some circles because the syndrome was more typical of enteric pathogens than of lentiviruses.

Definitive proof that $\operatorname{SIV}_{\mathrm{SMM} \cdot \mathrm{PB} j \mathrm{j}}$ causes this rapidly lethal disease is now established from the work of Dewhurst et al. ${ }^{2}$, who were able to generate fatal infections of pig-tailed macaques with virus derived from molecular clones. Three of six pigtailed macaques infected with cloned SIV $_{\text {SMM-PBji4 }}$ died eight days after infection; two animals succumbed on days 49 and 55 and one is still alive seven months later. Sequence analysis indicates, as expected, that SIV $_{\text {SMM-PBj } 14}$ is closely related to SIV $\mathrm{SMM}_{\mathrm{SM}}$ and shares less polynucleotide sequence homology with other SIVs and HIVs. Of note is a 23-base-pair duplication encompassing the single NF-KB enhancer element previously identified in the long terminal repeats of other SIVs.

What were the pathological findings in monkeys dying from this unusually fulminant and non-'lenti' (that is, rapid) lentivirus-induced disease? Philip Zack and colleagues (US Army Medical Research Institute of Infectious Diseases, Fort Detrick, Maryland) have infected juvenile rhesus, cynomolgus and pig-tailed macaques with SIV $_{\text {SMM.PBII4 }}$ and observed the rapidly fatal syndrome in all three species (P. Zack, personal communication). At autopsy, the most prominent feature was a two- to tenfold increase in the mass of many lymphoid organs; the spleen, mesenteric lymph nodes and the gut-associated lymphoid tissue (GALT) were the most severely affected. Diffuse infiltrates of lymphocytes, consisting of up to 50 per cent lymphoblasts, were present in perifollicular areas. In the intestine, multi-focal, patchy blunting of villi was seen in conjunction with hyperplasia of crypt epithelium; little if any ulceration of the epithelial layer was apparent.

According to Zack, high levels of virus expression are the hallmark of acute SIV $_{\text {SMM-PBi14 }}$-induced disease. From affected lymphoid tissue, $10^{4}-10^{5}$ infectious virions could be recovered. Viral antigens were synthesized in 20-50 per cent of lymphocytes and syncytial cells located in the lamina propria; by contrast, no viral protein was detected in the intestinal epithelial cells. Budding virus particles could readily be seen in lymphoblasts, macrophages and syncytia in the GALT.

\section{Immunological reaction}

The pathological changes affecting the gastrointestinal tract seem to be more typical of an immunologically mediated reaction than of a necrotizing infectious enterocolitis. In fact, a picture of infiltrating lymphocytes associated with villus atrophy and crypt hyperplasia is more characteristic of graft-versus-host disease ${ }^{4}$. It is not generally appreciated that the normal human intestinal mucosa is rich in T lymphocytes, plasma cells and cells of the monocyte-macrophage lineage. Although most of this lymphoid tissue is in the lamina propria, lymphocytes also reside in the epithelial layer and may constitute up to 39 per cent of the cells in this part of the mucosa. The migration of lymphocytes and lymphoblasts between these different intestinal compartments is extensive and tightly regulated. This mucosal lymphocyte population seems to play a large part in the renewal and differentiation of intestinal epithelial cells; alterations in intestinal $T$-cell function may, in fact, directly affect the proliferation of stem cells or early progenitor cells ${ }^{6}$.

With this as background, one can begin to speculate about how an $\operatorname{SIV}_{\text {SMM-PBj14 }}$ infection might kill monkeys rapidly, and about which viral genes or elements could be responsible for its virulent phenotype. First, it is clear that $\mathrm{SIV}_{\mathrm{SMM} \cdot \mathrm{PBj} 14}$ induces an acute and fulminant viral disease rather than the typical protracted immunodeficiency syndrome elicited by other primate lentiviruses. Circulating lymphocytes become an early target of SIV $\mathrm{SMM} \cdot \mathrm{PBj} 14$ and support numerous rounds of vigorous and highly efficient virus replication. Subsequently, a tidal wave of infected lymphocytes enter and become sequestered in lymphoid tissues associated with the gastrointestinal tract. Indeed, $\mathrm{SIV}_{\mathrm{SMM} \cdot \mathrm{PB} \mathrm{il}}$ can infect resting macaque lymphocytes in vitro and induce them to produce interleukin-2 (P. N. Fultz, personal communication). Assuming similar 
events occur in vivo, it is not difficult to imagine how SIV $_{\text {SMM-PBil4 }}$ might rapidly establish an extensive and spreading infection. But the migration of virus-producing lymphocytes to the GALT, and their apparent sequestration there, cannot yet be readily explained. Because SIV $_{\text {SMM.PBi14 }}$ can induce cytokines such as interleukin-2 in cultured lymphocytes, it would not be far-fetched to propose that infected lymphocytes and monocyte-macrophage populations in lymphoid structures associated with the gastrointestinal tract secrete cytokines which influence the normal mononuclear-cell traffic in these tissues, or directly affect gastrointestinal physiology. One or more of the SIV $_{\text {SMM.PBil4 }}{ }^{-}$ encoded proteins, such as an envelope protein that has undergone mutation, could elicit the 'inappropriate' production and/or release of such regulatory factors.

Monkeys infected with $\operatorname{SIV}_{\text {SMм- } \mathrm{PB} \mid 14}$ apparently have high levels of circulating tumour necrosis factor (TNF), which may be another reflection of aberrant virusinduced cytokine release. Increased TNF production is also associated with HIV-1 infection of primary human monocytes? The rapid clinical deterioration of SIV $_{\text {SMM-PBi!4 }}$-infected macaques, which develop profuse bloody diarrhoea and an associated metabolic acidosis, is reminiscent of Gram-negative septicaemia, presumably also mediated by TNF (ref. 8).

Lentiviruses characteristically cause a mild primary syndrome, usually followed by a prolonged and unremitting secondary disease which may last for months to years. In man, acute HIV infection is associated with a 'mononucleosis-like' disease accompanied by cervical, occipital, axillary or even generalized lymphadenopathy during this early phase of infection in 75 per cent of cases 9 . Interestingly, diarrhoea has been reported in a third of recently infected patients ${ }^{10}$. The acute disease caused by feline immunodeficiency virus also results in pronounced generalized lymphadenopathy which centres on the gastrointestinal tract ${ }^{11}$. Thus the disease induced in monkeys by $\mathrm{SIV}_{\mathrm{SMM}-\mathrm{PB} \mathrm{i} 14}$ could represent an unusually aggressive and

1. Kestler, H. et al. Science 248, 1109-1112 (1990)

2. Dewhurst, S., Embretson, J.E., Anderson, D.C., Mullins, J.I. \& Fultz, P.N. Nature 345, 636-640 (1990).

3. Fultz, P.N. et al. Aids Res. hum. Retroviruses 5, 397-409 (1989).

4. McDonald, G.B. \& Sale, G.E. in The Pathology of Bone Marrow Transplantation (eds Sale, G.E. \& Shulman H.M.) 77-103 (Year Book Medical, Chicago, 1984)

5. Ferguson, A. in Immunological Aspects of the Liver and the Gastrointestinal Tract (eds Ferguson, A. \& MacSween, R.N.M.) 152 (MTP, Lancaster, 1967).

6. MacDonald, T.T. \& Spencer, J. J. exp. Med. 167, 1341 1349 (1988).

. Merrill, J.E., Koyanagi, Y. \& Chen, I.S.Y. J. Virol. 63 4404-4408 (1989)

8. Tracy, K.J. et al. Science 234, 470-474 (1986).

9. Tindall, B., Cooper, D.A., Donovan, B. \& Penny, R. in Infectious Disease Clinics of North America - Medical Management of AlDS (eds Sande, M.A. \& Volberding, P.A.) 329-351 (W.B. Saunders, Philadelphia, 1988)

10. Cooper, D. A. et al. Lancet I, 537-540 (1985).

11. Yamamoto, J. K. et al. Am. J. vet. Res. 49, 1246-1258 (1988) highly aberrant primary lentivirus infection which efficiently and rapidly targets infected cells to lymphoid tissue in the digestive tract and results in death due to the loss of fluid and electrolytes.

The two new reports of infectious, disease-causing molecular clones of SIV also raise an interesting side issue pertaining to vaccine development. Fultz and her colleagues reported last year that asymptomatic sooty mangabey monkeys, previously infected with SIV $_{\text {SMM }}$, are completely protected from $\mathrm{SIV}_{\mathrm{SMM} \cdot \mathrm{PB}_{14}}$ induced disease ${ }^{3}$. The two sero-positive monkeys challenged with SIV $_{\text {SMM.PBjI4 }}$ did not die or show any signs of secondary disease. Although Desrosiers and his group have not carried out a similar experiment, they have isolated several molecular clones of SIV $\mathrm{VAC}_{\mathrm{MAC}}$, in addition to the disease-producing SIV $_{\text {MAC-239 }}$ clone, which give rise to persistent virus infections; so far, however, the avirulent clones have failed to induce immunodeficiency in inoculated macaques. One wonders whether avirulent variants of HIV that can replicate efficiently in vivo, and elicit similar protective immunity in man, might also be isolated or even constructed in the laboratory.

Malcolm A. Martin is in the Laboratory of Molecular Microbiology, National Institute of Allergy and Infectious Diseases, Bethesda, Maryland 20892, USA

\section{Blocks on the viral exit}

\section{Jonathan Weber}

Writing in News and Views nearly two years ago, David Baltimore ${ }^{1}$ coined the term 'intracellular immunization' to describe the prospect of using a dominant negative mutant viral gene to interfere with the replication of wild-type virus. The most attractive early application of this approach to cell protection is against HIV infection - both because of the pandemic nature of this lethal virus, and the possibility of relatively easy access to its target, the $\mathrm{CD}^{+}$peripheral blood mononuclear cells. Three candidate HIV genes in which mutations can be made to give a dominant negative phenotype have already been described: gag mutants, which probably interfere with HIV replication at the level of virus assembly ${ }^{2}$, and tat and rev transdominant mutants ${ }^{3,4}$. Now, on page 625 of this issue Buonocore and Rose ${ }^{5}$ describe a novel approach to 'intracellular immunization' using a CD4 mutant that is retained by the endoplasmic reticulum and that prevents transport of the HIV envelope proteins to the cell surface.

\section{Role of CD4}

That the CD4 molecule is the cellular receptor for HIV has been known since 1984, and soluble recombinant CD4 (srCD4) neutralizes HIV infection of target cells in vitro, and probably in vivo. But less attention has been paid to the role of CD4 in the transport of HIV env proteins and the production of mature viral particles. Asjo and colleagues ${ }^{6}$ showed that $\mathrm{CD} 4$ receptor density on diverse monocytoid cell line clones from the U937 parental line was associated with the quantity of infectious virus produced; Hoxie et al. $^{7}$, and subsequently Stevenson et al. ${ }^{8}$, demonstrated that down-regulation of CD4 post-HIV infection was associated with intracellular complexing of $\mathrm{CD} 4$ with HIV envelope protein. However, HIV env proteins can clearly be expressed in CD $4^{-}$cells, as shown by the Chinese hamster ovary cell line production of the HIV envelope glycoprotein gp120. The role of CD4 in the normal transport of gp120 requires further study.

Buonocore and Rose generated a mutant form of soluble CD4 (sCD4), which contains the sequence Lys-AspGlu-Leu (KDEL) at the C terminus; this sequence is a signal that can retain soluble proteins within the lumen of the endoplasmic reticulum". Constructs of sCD4KDEL were expressed in HeLa cells, with and without co-expression of gp120 and gp160. The sCD4-KDEL mutant prevented expression of gp120 on the cell surface, whereas normal SCD4 alone did not. In addition, the sCD4-KDEL mutant did not interfere with the normal surface expression of CD4 in the same cell. Using an assay for syncytium formation in HeLa CD4 $4^{+}$cells, Buonocore and Rose showed that sCD4-KDEL blocks syncytia formation after gp120/gp41 transfection. Theoretically, cells transfected with sCD4-KDEL should be protected from the consequences of HIV infection, while CD4 production and expression remains normal.

This approach to intracellular protection is elegant, and it avoids the use of dominant negative mutants based on viral genes which could lead to evasion of the block by further mutation. Any mutation of HIV env that prevents binding to SCD4-KDEL should reduce the binding of HIV to its receptor, and successful escape mutants from sCD4-KDEL should also be unable to infect $\mathrm{CD} 4^{+}$cells. But this study has not shown that wildtype HIV replication can be suppressed by sCD4-KDEL, nor that $T$ cells transfected with $s C D 4-K D E L$ are functionally normal. It remains possible that sCD4-KDEL could become saturated by 